\title{
Robust thermobarometry: constraints on the physical state of the mantle beneath the Kaapvaal craton
}

\author{
Karine S. Bégaudeau ${ }^{1}$ and Jean-Claude C. Mercier ${ }^{1,2}$ \\ ${ }^{1}$ UMR CNRS 6250 (LIENSS), ILE, University of La Rochelle, France \\ ${ }^{2}$ UMR CNRS 6112 (LPGN), University of Nantes, France
}

Ever since subcalcic garnet harzburgites have been described for the first time (Sobolev, 1977 ; Nixon et al., 1987; Schulze, 1994), the validity of thermobarometric estimates could be questionned, as well as any model for the infracratonic mantle. Indeed, all petrological as well as rheological/elastic models derived from the study of mantle xenoliths are essentially based on a thermometer using the Enstatitecomponent in clinopyroxene, that is on the clinopyroxene solvus toward orthopyroxene, a solvus highly sensitive to temperature with a $\mathrm{Ca}$ content in $\mathrm{M} 2$ as low as 0,54 in the highest-P-T xenoliths.

Formally, this thermometer is based on the transfer reaction $\operatorname{En}(\operatorname{cpx}) \leftrightarrow \mathrm{En}(\mathrm{opx})$. However, the equilibrium constant $X_{M g}^{M 2, c p x} / X_{M g}^{M 2, o p x}$ essentially depends on $X_{M_{g}}^{M 2, c p x}$, as natural $X_{M_{g}}^{M 2, o p x}$ poorly describes the opx solvus $(\mathrm{Ca}<0,055$ in the highest P-T xenoliths) to the point where some past thermometers have even neglected its effect and the reverse transfer reaction $X_{C a}^{M 2, o p x} / X_{C a}^{M 2, c p x}$ never been used. It follows that clinopyroxene must be strictly in equilibrium with the other phases at the time of sampling by the ascending magma for this thermometer to be warranted.

Actually, very few mantle peridotites do meet the minimum criteria for true chemical equilibrium to be likely, from both a theoretical standpoint and textural analyses. This is particularly true of the xenoliths brought up to the surface by kimberlites, especially the harzburgites (defined according to Streckeisen, 1973 ; that is a peridotite with less than $5 \% \mathrm{cpx}$ ) which make up to $90 \%$ of such xenoliths and which are largely regarded as being representative of the Archean lithosphere ;

A priori models for the origin of such rocks are not consistent with the concept of subsolidus equilibrium.

From a rheologial standpoint, the negligeable strainrate / medium stress conditions that they may represent are unlikely to have yielded the mechanical twinning energetically necessary for the transfer reactions to proceed through exsolution, that is to achieve and maintain equilibrium throughout millions to billions of years. This is even more the case for rocks in which olivine is the dominant modal phase, as its lower critical stress makes it flow so more easily that it behaves as a matrix passively carrying the other phases. In any case, if some unsuspected process had allowed mutual exsolution, or if there had been some minor $\mathrm{Ca}-\mathrm{Mg}$ exchange between the pyroxene phases through some dual resorption/growth process, this could have only affected adjacent grains, with the likely implication of inducing some phase heterogeneity. No evidence of the latter has ever been observed, and clinopyroxene is not texturally associated to orthopyroxene.

Of course, one may call upon solidus equilibrium, in which case equilibrium between the phases would be achieved through a minor liquid film at grain boundaries ( $\geq 0.7 \mathrm{~mm}$ according to observations on Hawaiian dunite cumulates ; Mercier, 1988), which can be an efficient transfer medium. Such an explaination however suggests that the overall lithospheric Archean mantle would still be in dry-solidus conditions, which is not consistent with the melting-experiment data, neither in terms of P-T conditions, nor in terms of the order of disappearance of the phases (garnet before clinopyroxene; Kinzler \& Grove, 1999). This also excludes any model considering that the harzburgites would represent Archean cumulates and their clinopyroxene compositions, those of a quenched phase representative of cristallization conditions.

One may alternatively consider that the harzburgites have been at least partially reequilibrated through pervasive metasomatism at various times, since when they reached a modal composition closed to that observed. In this case, depending on the sample, metasomatism could be cryptic and/or modal, with all the harzburgites deriving from primordial subcalcic facies devoid of clinopyroxene.

In many samples from Thaba Putsoa and Letseng, the clinopyroxene crystals observed in series of thin sections, either were in symplectites related to garnet, or occurred as small dispersed grains clearly of PIC or MARID metasomatic origin, i.e. crystallized from, or reequilibrated with, melts linked to Group I or Group II 
kimberlites (Coussaert et al., 2003 ; Coussaert, 2005), and mostly in relation to healed fractures. In some samples, orthopyroxene had significant « cryptic » rims (i.e. not overgrowths) indicative of re-equilibration with a liquid, without any textural/modal evidence of metasomatism.

Such liquids could have thus crystallized all the observed clinopyroxene, or, at least, would have completely reequilibrated this low melting-point phase. It follows that all models (thermal, rheological, etc.) based on the so-called two-pyroxene thermometry are presently un-warranted. Yet, they may be correct within the analytical error if the phase crystallized or re-equilibrated near/at the subsolidus temperature of the host rock due to its thermal inertia relative to minor liquids passing through, but this has not yet be proven. As for the other thermobarometers not involving clinopyroxene (Fe-Mg exchanges, Ca-transfer between opx and ol), they are much less experimentally constrained than the clinopyroxene solvus.

According to the above considerations, it seemed worth trying to work out the wealth of experimental data on the orthopyroxene-clinopyroxene couple, in a attempt to model their mutual solubility in various systems (up to the natural one) by simultaneously solving both equation by inversion, without any a priori forcing of the model. In spite of the very poor relative precision on the orthopyroxene composition in experiments, an improved solution model could still be discriminant enough to decide whether clinopyroxenes in natural harburgite are significantly out of equilibrium, or if the eventual offset remains in the a-posteriori error bracket inferred from the inversion.

In a general inversion procedure, individual errors on the prior experimental data and prior model parameters are minimized simultaneously to get the best possible law describing the experimental dataset (e.g. Bertrand and Mercier, 1985). However, for the first time, the procedure used (Bégaudeau et al., in preparation) identifies by itself which parameters of the theoretical equations are significant, thereby providing a unique and robust solution independent of any prior assumption, inasmuch are both transfer reactions (for the En and the Di components) are truly solved simultaneously.

The theoretical approach is that of statistic thermodynamics with the non-ideal behaviors and the interactions among elements within sites and across sites being strictly formalized through Margules parameters. The starting equations include all the parameters inferred from statistical thermodynamics by combining these theoretical concepts and cristallographic constraints. However, the available experimental datasets are more and more degraded as the system evolves from the simplest CMS case to natural compositions. Not only are fewer experimental data available, but, as components are added, achieving true reversals becomes unrealistic, financially and time-wise. Fortunately, this can be easily taken into account in the inversion procedure since the Margules terms are purely additive.

Inversion of the available CMS data provides a robust model (Fig. 1) describing clinopyroxene as a classical asymetric solution, but a non-ideality term also showed up for orthopyroxene while in all of the (few) former models including such a term (e.g. Lindsley and Dixon, 1976; or Gasparik, 1990), it was only an a-priori guestimate. We also confirm the early work of Bertrand and Mercier (1985) about the nature of the Margules parameters : only the temperature terms are definitely significant, which had already been suggested by Coussaert (2005), but through a mathematical approach which had to be fully revised.

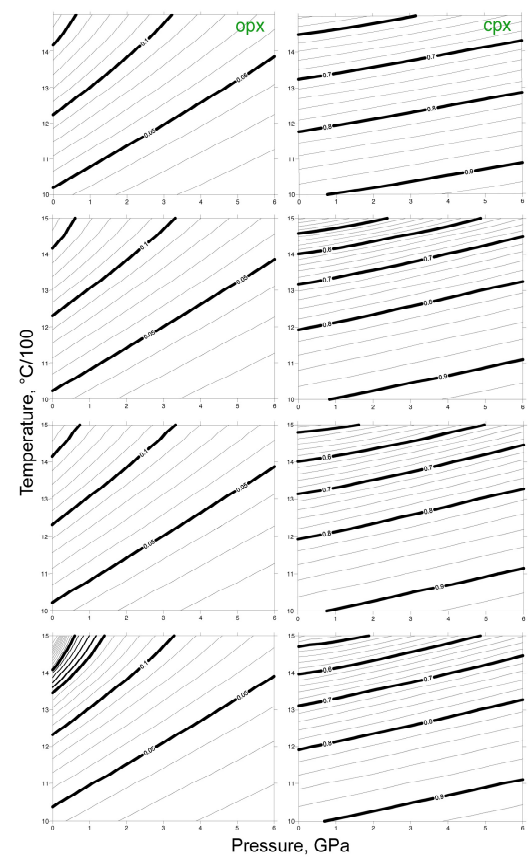

Figure 1. CMS solution models as solvi projection in P-T space. From top to bottom, opx/cpx solutions are: ideal/ideal, ideal/symetric, ideal/asymetric, symetric/ asymetric

When increasing the system complexity, the inversion tends to yield global correction factors to the simpler systems. Despite the fact that eye inspection of some graphs may suggest a virtual lack of correlation between the experimental and recalculated P-T conditions, and even more so for $\mathrm{P}$ (Fig. 2), the significant Margules parameters for the only Al-related excess terms, for example, are still very well contrained as shown in Fig. 3. Indeed, three distinct behaviors are observed. The two curves identified as Wse represent very stable and obviously significant Margules parameters defining the excess entropies for the En reaction due to addition of $\mathrm{Al}$ to the CMS system. The curves identified as Wsd represent the $\mathrm{W}$ parameters of excess entropies for the Di reaction. As usual, the poor precision on orthopyroxene compositions creates a noise which makes the system unstable at low variances. Above 100, however, the 
present inversion procedure provides $\mathrm{W}$ values as much significant as those for the En reaction. As for the other parameters (Wu's, Wv's), their a-posteriori values would clearly be dependent on the a-priori variance for any reasonable value.

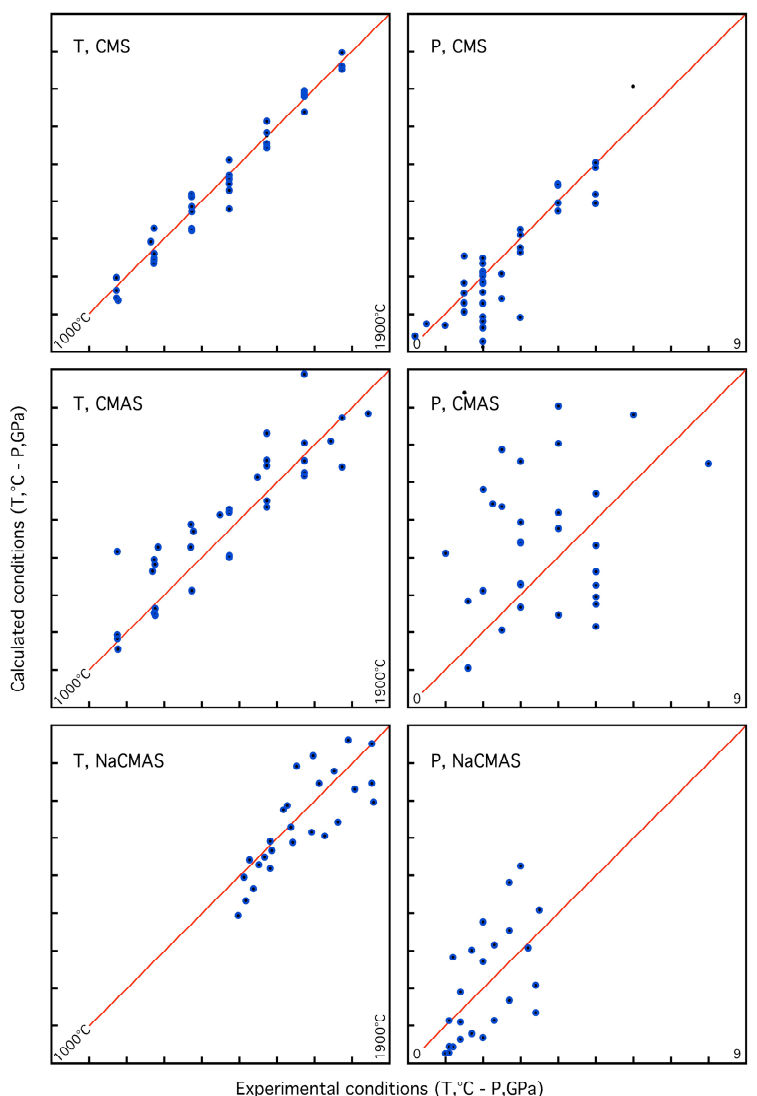

Figure 2. Calculated versus experimental P-T conditions showing the progressive relative degradation from CMS to NaCMAS.

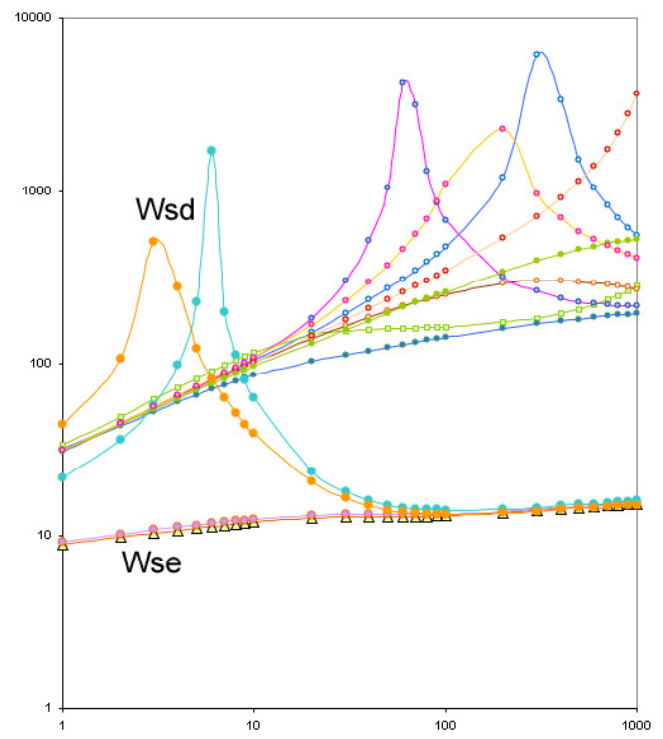

Figure3. Precision on the Margules parameters (standard deviation / model value) for the excess terms modelling the addition of Al to the CMS system, as a function of a-priori fixed variances.
Our final equations further adjusted for $\mathrm{Cr}$ and $\mathrm{Fe}$, and tested on the natural system, hence provide a robust self-sufficient two-pyroxene geothermometer. Application to a depth-representative set of spinel and/or garnet peridotites from Letseng and Thaba Putsoa, Lesotho, and a few other representative localities, will indicate whether or not the usual hypothesis of opxcpx-gt equilibrium on which models are based, does really hold. The final results are presented at the Conference, with the thermodynamic tools developped made available on-line.

\section{References}

Bertrand, P., Mercier, J-C.C., 1985. The mutual solubility of coexisting ortho- and clinopyroxene: toward an absolute geothermometer for the natural system? Earth Planetary Science Letters 76, 109-122

Coussaert, N., 2005. Evolution apparente du degré d'équilibre $\mathrm{du}$ manteau archéen par métasomatisme et déformation: exemple des nodules mantelliques du Lesotho. Ph.D. thesis, Université Libre de Bruxelles \& Université de La Rochelle, $321 \mathrm{pp}$.

Coussaert, N., Grégoire, M., Mercier, J-C.C., Bell, D., Demaiffe D., Le Roex A., André L., 2003a. The origin of clinopyroxene in cratonic mantle. 8IKC Extended Abstract Volume, FLA0383

Coussaert, N., Mercier, J-C.C., Demaiffe, D., André, L., 2003a. Equilibrium conditions revisited for Lesotho kimberlites. 8IKC Extended Abstract Volume, FLA0266

Kinzler, R.J., Grove, T.L., 1999. Origin of depleted cratonic harzburgite by deep fractional melt extraction and shallow olivine cumulate infusion. In: The J.B. Dawson volume, 7IKC Proceedings, J.J. Gurney et al. Eds, Capetown. 437-443.

Mercier, J-C.C., 1988. Données nouvelles sur les enclaves mantelliques d'Hawaii. Supplément Bulletin de Minéralogie 111 [12/88].

Nixon, P.H., van Calsteren, W, Boyd, F.R., Hawkesworth, C.J., 1987. Harzburgites with garnets of diamond facies from southern African kimberlites, In: Nixon P.H (Ed.), Mantle Xenoliths, Wiley \& Sons Ltd, Chichester, 533-537

Schulze, D.J., 1994. Abundance and distribution of low-Ca garnet harzburgites in the subcratonic lithosphere of southern Africa. In: Kimberlites, related rocks and mantle xenoliths. 5IKC Proceedings. CPRM, Rio de Janeiro. 327-335.

Sobolev, N.V., 1977. Deep-seated inclusions in kimberlites and the problem of the composition of the upper mantle. American Geophysical Union (Ed.), Washington, D.C., 279 pp

Streckeisen, A., 1973. Plutonic rocks. Classification and nomenclature recommended by the IUGS Subcommission on the Systematics of Igneous Rocks. Geotimes 18: 26-30 